\title{
Comparisons between Orange- and Green- fleshed Non-netted and Orange-fleshed Netted Muskmelons: Antioxidant Changes following Different Harvest and Storage Periods
}

\author{
D. Mark Hodges ${ }^{1}$ \\ Atlantic Food and Horticulture Research Centre, Agriculture and Agri-Food Canada, Kentville, N.S., \\ B4N 1J5 Canada
}

Gene E. Lester

Kika de la Garza Subtropical Agricultural Research Center, USDA-ARS, Weslaco, TX 78596

AdDITIONAL INDEX WORDS. ascorbic acid, ß-carotene, human nutrition, oxidative stress, phenolics, postharvest, quality

\begin{abstract}
Aвstract. The consumption of netted muskmelons (Cucumis melo $\mathrm{L}$. Reticulatus group) has raised health concerns due to pathogenic bacteria attaching to sites on the netted rind inaccessible to sanitation. The purpose of this study was to compare 1) the enzymic and nonenzymic antioxidant capacity between representative cultivars of netted muskmelon and both green- and orange-fleshed honey dew muskmelons during storage for 17 days and 2) levels of non-nutrient phytochemicals between these genotypes in consideration of ultimately substituting netted orange-fleshed with nonnetted orange-fleshed muskmelon. Netted muskmelon ('Cruiser'), green-fleshed ('Honey Brew'), and orange-fleshed ('Orange Dew') muskmelons were harvested in Texas at the beginning (21 May) and at the end (11 June) of the production season in 2004. Fruit were analyzed immediately (day 0 ) or stored simulating retail conditions for 7 or 14 days at $7^{\circ} \mathrm{C}$ and $95 \% \pm 2 \%$ relative humidity plus 3 days at $21^{\circ} \mathrm{C}$. Both 'Orange Dew' and 'Honey Brew' non-netted cultivars evinced similar and less lipid peroxidation, and hence postharvest senescence, during the 17-day storage period than the netted muskmelon 'Cruiser'. In comparison with 'Cruiser', 'Orange Dew' generally exhibited higher concentrations of $\beta$-carotene and phenolics and, with few exceptions, higher activities of the antioxidant enzymes ascorbate peroxidase (AsPX), monodehydroascorbate reductase (MDHAR), dehydroascorbate reductase (DHAR), catalase (CAT), guaiacol peroxidase (POX), and superoxide dismutase (SOD). Higher AsPX and SOD activities in both 'Orange Dew' and 'Honey Brew' appear to confer a greater resistance to lipid peroxidation in these muskmelon genotypes than to the netted 'Cruiser'. 'Orange Dew' also appears to be a healthier food choice not only due to its lack of a netted rind which could potentially harbour human illness-related pathogens, but also that it is superior to both 'Cruiser' and 'Honey Brew' in overall beta-carotene and phenolic levels.
\end{abstract}

Among the different fruit and vegetables associated with food borne illness, netted muskmelon has consistently tested positive for Salmonella Lignières (Castillo et al., 2004), Shigella Chatellani \& Dawson (U.S. Food and Drug Administration, 2003), and Escherichia coli O157:H7 (Del Rosario and Beuchat, 1995), and has been associated with large outbreaks of human illness (more than 25,000 individual cases since 1990) in the United States and Canada (Castillo et al., 2004), and in 2001 and 2002 two cases of human death (Canadian Food Inspection Agency, 2003). Survival of human illness pathogens on netted muskmelon following surface pasteurization (Annous et al., 2004; Ukuku, 2004) is attributed to inaccessible sites on the rind such as the netting (Beuchat and Scouten, 2004). Replacing netted, orange-fleshed muskmelon with a suitably phytonutrient

Received for publication 16 May 2005. Accepted for publication 16 Aug. 2005. Atlantic Food and Horticulture Research Centre Contribution No. 2303. This research was funded in part by Agriculture and Agri-Food Canada (D.M.H.) and the USDA-ARS under CRIS No. 6204-43000-014-00D to G.E.L. The valuable technical assistance of Robert D. Meyer (USDA-ARS, Weslaco, Texas), Vivian E. Willmets and Michele L. Elliot (both Agriculture and Agri-Food Canada, Kentville, N.S.) is gratefully acknowledged. The authors wish to thank Drs. John DeLong and Charles Forney, Atlantic Food and Horticulture Research Centre, Agriculture and Agri-Food Canada, Kentville, N.S., for critical review of this manuscript. Use of company or product names by the USDA or Agriculture and Agri-Food Canada does not imply approval or recommendation of the product to the exclusion of others that may be suitable.

'To whom reprint requests should be addressed. Email: HodgesM@agr.gc.ca dense non-netted, orange-flesh genotype such as orange-fleshed honey dew (C. melo L. Inodorus group) would greatly reduce the incidence of food borne illness associated with netted fruit. Non-netted, orange-fleshed honey dew fruit, such as 'Orange Dew', are commercially grown in limited quantities in the U.S. However, little is known regarding the human health-promoting phytochemicals or the antioxidant capacity of these genotypes compared to netted muskmelon cultivars. Moreover, enzymatic antioxidant capacity in orange-fleshed honey dew muskmelon associated with postharvest storage life has not been addressed. The current postharvest shelf-life of a typical green-fleshed honey dew muskmelon is 3-4 weeks (Edwards and Blennerhassett, 1990) and $\approx 10$ to $14 \mathrm{~d}$ for netted muskmelon (Lester, 1988). It is unknown if non-netted orange-fleshed honey dew muskmelon cultivars have a postharvest storage life similar to those of green-fleshed honey dew or orange-fleshed netted muskmelons. Additionally, it is unknown what effect the environment plays on antioxidant capacities in melon genotype fruit harvested at the beginning vs. the end of the production season.

Oxidative stress has been associated with postharvest quality losses of fresh fruit and vegetables (Hodges et al., 2004). Active oxygen species (AOS) such as superoxide $\left(\mathrm{O}_{2}^{-}\right)$and the hydroxyl radical $(\cdot \mathrm{OH})$ play significant roles in lipid peroxidation, polysaccharide cleavage, and in both nucleic acid and protein degradation (Hodges, 2003). Past work has shown that capacities of certain antioxidants are important in maintaining postharvest quality 
in spinach (Spinacia oleracea L.) (Hodges et al., 2001; Hodges and Forney, 2003), apples (Malus ×domestica Borkh.) (Barden and Bramlage, 1994), pears (Pyrus communis L.) (Vanoli et al.,1995), pepper fruit (Capsicum annuum L.) (Jiménez et al., 2003), broccoli (Brassica oleracea L. Italica group) (Toivonen and Sweeney, 1998; Yamauchi and Kusabe, 2001), potato (Solanum tuberosum L.) seed tubers, (Zabrouskov et al., 2002), and nonnetted muskmelon (Lacan and Baccou, 1998). In addition, human epidemiological evidence has linked intake of fruit and vegetables with lower incidences of cancer, cardiovascular disease, immune system decline, and certain neurological disorders (Huang et al., 2005), all of which have been associated with oxidative damage (Hodges and Kalt, 2003); it is the antioxidative capacity in fruit and vegetables that has been identified as one of the major mechanisms contributing to human health maintenance (Hodges and Kalt, 2003; Olsson et al., 2004; Vinson et al., 2001).

Enzymic and nonenzymic antioxidants represent an important mechanism in AOS scavenging (for reviews, see Blokhina et al., 2003; Lurie, 2003). Superoxide dismutase [SOD (EC 1.15.1.1)] catalyzes the dismutation of $\mathrm{O}_{2}^{-}$to $\mathrm{H}_{2} \mathrm{O}_{2}$. Both catalase [CAT (EC 1.11.1.6)] and ascorbate peroxidase [AsPX (EC 1.11.1.11)] eliminate $\mathrm{H}_{2} \mathrm{O}_{2}$, with ascorbate (vitamin C) being oxidized during the latter reaction catalyzed by AsPX. Ascorbate is re-reduced directly through activities of monodehydroascorbate reductase [MDHAR (EC 1.6.5.4)] and dehydroascorbate reductase [DHAR (EC 1.8.5.4)] and indirectly through activity of glutathione reductase [GR (EC 1.6.4.2)]. Peroxidases [POX (EC 1.11.1.7)] also convert $\mathrm{H}_{2} \mathrm{O}_{2}$ to $\mathrm{H}_{2} \mathrm{O}$, though they can form $\mathrm{O}_{2}{ }^{-}$and $\mathrm{H}_{2} \mathrm{O}_{2}$ in a complex reaction involving $\mathrm{NADH}$ oxidation.

Not only can ascorbate reduce $\mathrm{H}_{2} \mathrm{O}_{2}$ in the AsPX-catalyzed reaction described above, but this compound can also react directly with the AOS $\mathrm{O}_{2}^{-}, \cdot \mathrm{OH}$ and singlet oxygen $\left({ }^{1} \mathrm{O}_{2}\right)$ (Hodges and Forney 2003), and may play a role in the regeneration of $\alpha$-tocopherol (Horemans et al., 2000). One of the primary antioxidant functions of $\beta$-carotene, a lipid soluble antioxidant precursor to vitamin $\mathrm{A}$, is to quench ${ }^{1} \mathrm{O}_{2}$ (Knox and Dodge, 1985). The phenolic class of phytochemicals, which include flavonoids, tannins, hydroxycinnamate derivatives, and lignin, also possess antioxidative properties due to their high reactivity as hydrogen or electron donors, their ability to chelate proxidant transition metal ions such as $\mathrm{Fe}^{3+}$ and $\mathrm{Cu}^{3+}$, and their free radical chainbreaking function (Blokhina et al., 2003; Hodges and Kalt, 2003; Proestos et al., 2005). Studies have suggested that fruit extracts and/or isolated phenolics are associated with anti-cancer, antiinflammatory, cardioprotective, and neuroprotective properties (Hodges and Kalt, 2003).

The purpose of this study was to compare 'Orange Dew', a representative orange-fleshed honey dew melon, with 'Cruiser,' a netted orange-fleshed muskmelon, for 1) enzymatic and nonenzymatic (human nutritional) antioxidant capacities, 2) stability of antioxidant capacity over the commercial harvesting period, and 3 ) postharvest senescence (following $17 \mathrm{~d}$ of simulated commercial/retail storage). For comparative purposes, fruit of the green-fleshed honey dew muskmelon 'Honey Brew' were also included in this study.

\section{Materials and Methods}

Plant material. Fully abscised fruit of orange-fleshed, netted muskmelon ('Cruiser'), orange-fleshed, non-netted honey dew ('Orange Dew'), and green-fleshed, non-netted honey dew ('Honey Brew') melons, free of defects, were hand-harvested from Starr Produce Co. (Rio Grande City, Texas). All fruit were collected from the field by $0900 \mathrm{HR}$, at the beginning of the commercial harvest season (21 May) and 3 weeks later at the end of the 2004 season (11 June). Fruit were washed in $0.02 \%$ sodium hypochlorite for $30 \mathrm{~s}$, rinsed in tap water, and then randomized into lots for storage treatment. Fruit were assessed the day of harvest or after storage for 7 or $14 \mathrm{~d}$ at $7{ }^{\circ} \mathrm{C}$ and $95 \% \pm 2 \% \mathrm{RH}$ plus $3 \mathrm{~d}$ at $21{ }^{\circ} \mathrm{C}$ to simulate commercial storage plus retail display temperatures.

All fruit were chilled to $4{ }^{\circ} \mathrm{C}$, washed with distilled water, the epidermis removed with a vegetable peeler, and the polar-ends (totaling two-thirds of the fruit) removed and discarded. Wedges of the remaining equatorial-region mesocarp tissue, devoid of seeds and integument tissue, were pureed in a food processor (Quick 'N Easy; Black and Decker, Towson, Md.) using 3- to 5-s pulses. Tissue samples were assayed fresh for enzymes, frozen (liquid nitrogen, then stored at $-80{ }^{\circ} \mathrm{C}$ ) for compounds, and lyophilized (following freezing in liquid nitrogen) for total antioxidants.

Metabolite asssays. Ascorbic acid and dehydroascorbic acid were extracted from frozen tissue and determined according to the procedure of Hodges et al. (2001) and reported in this study as total ascorbate.

ß-carotene was extracted under low light conditions from lyophilized tissue $(0.020 \mathrm{~g})$ using ice-cold heptane $(1.0 \mathrm{~mL})$ plus 0.5 mL internal standard [trans Apo- $8^{\prime}$-carotene $\left(40 \mu \mathrm{g} \cdot \mathrm{mL}^{-1}\right)$; Sigma Chemical Co., St. Louis] according to the modified procedure of Koch and Goldman (2004). The internal standard stock solution was made by dissolving Apo-8'-carotene in $1.0 \mathrm{~mL}$ methanol then bringing to volume $(250 \mathrm{~mL})$ with heptane before storage in the dark at $-20^{\circ} \mathrm{C}$. This sample-internal standard mixture was vortexed for $2 \mathrm{~min}$, then centrifuged at $3000 \mathrm{~g}_{\mathrm{n}}$ for $10 \mathrm{~min}$ at 0 ${ }^{\circ} \mathrm{C}$. One milliliter of the supernatant was removed and $1.5 \mathrm{~mL}$ fresh cold heptane was added to the pellet, vortexed for 2 min then centrifuged as above. A second $1.0 \mathrm{~mL}$ of the supernatant was removed and $1.0 \mathrm{~mL}$ cold heptane was added to the pellet, vortexed for $2 \mathrm{~min}$ then centrifuged as above. A third $1.0 \mathrm{~mL}$ of the supernatant was removed, the supernatants were pooled and a 1.0-mL aliquot was passed through a 0.2-mm nylon Millex-LCR 13 filter (Millipore Corp., Bedford, Mass.) and stored in the dark at $-20{ }^{\circ} \mathrm{C}$ until high-performance liquid chromatograph (HPLC) determination. Twenty microliters of each extract was injected into an HPLC system (Agilent Technologies, New Castle, Del.) equipped with a Discovery $\mathrm{C} 18,5-\mu \mathrm{m}$ column $(150 \times 4.6 \mathrm{~mm})$ and a C18 guard column $(20 \times 4.0 \mathrm{~mm}$; Supelco, Bellefonte, Pa. $)$. $\beta$-carotene was separated in a mobile phase of $100 \%$ methanol at a flow rate of $2.0 \mathrm{~mL} \cdot \mathrm{min}^{-1}$ and detected at $454 \mathrm{~nm}$.

Malondialdehyde content was determined on $2.0 \mathrm{~g}$ of fresh tissue using the TBARS procedure of Hodges et al. (1999). Phenolic levels in $2.0 \mathrm{~g}$ of fresh tissue were assayed using the Folin-Ciocalteu method (Singleton and Rossi, 1965). Results are expressed as gallic acid equivalents $\left(\mathrm{mg} \cdot \mathrm{g}^{-1} \mathrm{FW}\right)$. Protein content was determined using the Bio-Rad assay (Bio-Rad Lab. Hercules, Calif.) based on the method of Bradford (1976).

ENZYME ASSAYS. Activities of AsPX, CAT, DHAR, GR, MDHAR, POX, and SOD were analyzed in $15 \mathrm{~g}$ of fresh tissue as described in Lester et al. (2004).

Total antioxidant assay. Both lipophilic and hydrophilic antioxidants were analyzed using randomly methylated $B$-cyclodextrin (RMCD) as a solubility enhancer, 2,2' -azobis (2-amidinopropane) dihydrochloride (AAPH) as a peroxyl generator and 6hydroxy-2,5,7,8-tetramethylchroman-2-carboxylic acid (Trolox) as a standard according to Prior et al. (2003). 
Statistical analyses. Analysis of variance (ANOVA) using the general linear model procedures of SAS (SAS Institute, Cary, N.C.) was conducted as a three-factor design with cultivar, harvest time, and storage duration as the main factors (Table $1)$. Duncan's multiple range tests $(P \leq 0.05)$ were performed to evaluate the significance of differences between dependent variable means. Data are the average of five single-fruit replications per harvest.

The enzyme and MDA means for cultivar, harvest time, and storage duration were extracted from the ANOVA and submitted to a principal component analysis (PCA) using GenStat (release 8.1; VSN International Ltd., Hemel Hempstead, England). The PCA loadings produced three major components accounting for $71 \%$ of the enzyme and MDA variation by treatments.

\section{Results}

Metabolites. No significant replicate effect was detected for any of the variables measured (Table 1). Levels of MDA $\left(\mathrm{nmol} \cdot \mathrm{g}^{-1} \mathrm{FW}\right)$, the estimator of lipid peroxidation and indicator of senescence was significant for cultivar, harvest and storage period, and their interactions. Although MDA concentrations increased in all cultivars during $17 \mathrm{~d}$ of storage, 'Cruiser' exhibited the highest level of lipid peroxidation while 'Orange Dew' exhibited the lowest level, which was similar to 'Honey Brew' (Table 2). MDA content, in general, was higher in all fruit at the end of the harvest season versus the start.

Total ascorbate concentrations ( $\mu \mathrm{mol} \cdot \mathrm{g}^{-1} \mathrm{FW}$ ) were significantly different for cultivars, harvest and storage periods, but not for their interactions (Table 1). Total ascorbate content declined during storage in the non-netted green- and orange-fleshed muskmelon, but not in the netted muskmelon (Table 2). However, the only absolute difference between cultivars occurred on day 17 of storage of early harvest fruit, where total ascorbate levels in the non-netted muskmelon were less than in the netted cultivar, and on day 0 of late harvest fruit, where 'Honey Brew' exhibited lower total ascorbate concentration.

ß-carotene $\left(\mathrm{nmol} \cdot \mathrm{g}^{-1} \mathrm{FW}\right)$ was significant only for cultivars; there were no effects of harvest period, storage period, or their interactions (Table 1). B-carotene was not detected in 'Honey Brew' (Table 2.). Levels of this lipophilic compound were always higher in 'Orange Dew' compared to the netted melon 'Cruiser,' regardless of storage duration or time of harvest.

No differences in phenolic levels, expressed as gallic acid equivalents $\left(\mathrm{mg} \cdot \mathrm{g}^{-1} \mathrm{FW}\right)$ were observed between early and late harvests (Table 1). Phenolic levels increased in all cultivars during storage, and, with the exception of non-netted 'Honey Brew' on day 10 of the early harvest and netted 'Cruiser' on day 17 of the late harvest, phenolic levels were generally higher on days 10 and 17 in non-netted 'Orange Dew' (Table 2). No difference in phenolic concentrations between the three cultivars was observed on day 0 .

Antioxidant Enzymes. No significant differences in activities of any of the antioxidant enzymes were noted between replicates, and no differences between harvests were observed for activities of the enzymes MDHAR, DHAR, GR, and CAT (Table 1). Activities of AsPX $\left(\mathrm{mmol} \cdot \mathrm{min}^{-1} \cdot \mathrm{g}^{-1} \mathrm{FW}\right)$ were higher in 'Orange Dew' compared to 'Cruiser' (Table 3). Both 'Honey Brew' and 'Orange Dew' exhibited significantly higher AsPX activities than 'Cruiser' on day 17.

Activities of MDHAR ( $\left.\mathrm{mmol} \cdot \mathrm{min}^{-1} \cdot \mathrm{g}^{-1} \mathrm{FW}\right)$ increased during storage for all cultivars harvested at the end of the season; similar results were observed in early harvest 'Honey Brew' and 'Orange Dew' fruit (Table 3). With the exception of day 0 fruit from the early harvest and day 17 fruit from the late harvest, 'Orange Dew' exhibited the highest MDHAR activities. DHAR $\left(\mathrm{mmol} \cdot \mathrm{min}^{-1} \cdot \mathrm{g}^{-1} \mathrm{FW}\right)$ activities, similar to those of AsPX and MDHAR, were higher in 'Orange Dew' compared to 'Cruiser' during storage. By day 17, 'Orange Dew' had higher DHAR activities than either 'Cruiser' and 'Honey Brew,' activities of which were not significantly different from one another.

Higher activities of GR $\left(\mathrm{mmol} \cdot \mathrm{min}^{-1} \cdot \mathrm{g}^{-1} \mathrm{FW}\right)$ occurred in 'Orange Dew' fruit compared to the two other cultivars on days 0,10 , and 17 of the late harvest; no difference were noted in GR activities between 'Cruiser' and 'Honey Brew' (Table 4). However, in fruit from the beginning of the harvest season, 'Cruiser' and 'Orange Dew' exhibit higher GR activities than 'Honey Brew.'

Activities of CAT ( $\mathrm{mmol} \cdot \mathrm{min}^{-1} \cdot \mathrm{g}^{-1} \mathrm{FW}$ ) were also significantly higher in 'Orange Dew' as compared to 'Cruiser' on day 10, and were higher in 'Orange Dew' fruit then the other cultivars on day 17 (Table 4). There were no differences in activities of CAT between 'Cruiser' and 'Honey Brew' on days 10 and 17. Activities of this enzyme, although initially high, declined in 'Honey Brew' during the 17-d storage period.

POX activities ( $\left.\mathrm{mmol} \cdot \mathrm{min}^{-1} \cdot \mathrm{g}^{-1} \mathrm{FW}\right)$ were higher in 'Orange Dew' fruit on all storage days in the start of the harvest season; no differences were noted in POX activities between 'Cruiser' and 'Honey Brew' (Table 4). However, 'Orange Dew' was the only cultivar where POX activity declined during storage, whereas activities of this enzyme remained constant in the other cultivars during the 17-d storage period. In end of the season harvested fruit, 'Orange Dew' and 'Honey Brew' had higher POX activities than 'Cruiser,' and no overall change in activity was noted following storage for any of the cultivars.

SOD activities $\left(\mathrm{mmol} \cdot \mathrm{min}^{-1} \cdot \mathrm{g}^{-1} \mathrm{FW}\right)$ were higher in 'Orange Dew' than 'Cruiser' on days 10 and 17; no differences in activities

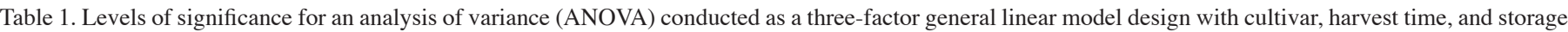
duration as the main factors for concentrations of malondialdehyde (MDA), ascorbate (AsA), ß-carotene (ß-carot.), phenolics, total hydrophilic antioxidants (THA) and total lipophilic antioxidants (TLA) and activities of ascorbate peroxidase (AsPX), monodehydroascorbate reductase (MDHAR), dehydroascorbate reductase (DHAR), glutathione reductase (GR), catalase (CAT), guaiacol peroxidase (POX), and superoxide dismutase (SOD).

\begin{tabular}{|c|c|c|c|c|c|c|c|c|c|c|c|c|c|}
\hline Source & MDA & AsA & ß-carot. & THA & TLA & Phenolics & AsPX & MDHAR & DHAR & GR & CAT & POX & SOD \\
\hline Rep & NS & NS & NS & NS & NS & NS & NS & NS & NS & NS & NS & NS & NS \\
\hline Cultivar (C) & 0.0001 & 0.0001 & 0.0001 & 0.0001 & 0.04 & 0.0001 & 0.001 & 0.001 & 0.0001 & 0.01 & 0.01 & 0.001 & 0.0001 \\
\hline Harvest (H) & 0.0001 & 0.001 & NS & NS & 0.0001 & NS & 0.01 & NS & NS & NS & NS & 0.01 & 0.0001 \\
\hline Storage (S) & 0.0001 & 0.0001 & NS & 0.001 & 0.001 & 0.0001 & 0.01 & 0.001 & 0.001 & NS & 0.001 & 0.01 & 0.0001 \\
\hline $\mathrm{C} \times \mathrm{H}$ & 0.0001 & NS & NS & NS & NS & NS & 0.001 & NS & NS & NS & NS & 0.001 & 0.0001 \\
\hline $\mathrm{C} \times \mathrm{S}$ & 0.0001 & NS & NS & NS & NS & NS & NS & 0.01 & 0.01 & NS & 0.001 & NS & 0.0001 \\
\hline $\mathrm{H} \times \mathrm{S}$ & 0.0001 & NS & NS & NS & 0.0001 & 0.01 & NS & NS & NS & NS & NS & NS & 0.0001 \\
\hline $\mathrm{C} \times \mathrm{H} \times \mathrm{S}$ & 0.0001 & NS & NS & NS & NS & NS & NS & NS & NS & NS & NS & 0.01 & 0.0001 \\
\hline Error mean square & NS & NS & NS & NS & NS & NS & NS & NS & NS & NS & NS & NS & NS \\
\hline
\end{tabular}


Table 2. Malondialdehyde (MDA), total ascorbate, $\beta$-carotene, and phenolic contents (expressed as gallic acid equivalents) in orange-fleshed cantaloupe cultivar Cruiser, green-fleshed honeydew cultivar Honey Brew and orange-fleshed honeydew cultivar Orange Dew fruits from plants at the beginning (Early) of the harvest season and from the same plants at the end of the harvest (Late) season and following 0,10 ( $7 \mathrm{~d}$ at 10 ${ }^{\circ} \mathrm{C}$ and $95 \%+2 \% \mathrm{RH}$ plus $3 \mathrm{~d}$ at $\left.21^{\circ} \mathrm{C}\right)$, or $17 \mathrm{~d}\left(14 \mathrm{~d}\right.$ at $10{ }^{\circ} \mathrm{C}$ and $95 \%+2 \% \mathrm{RH}$ plus $3 \mathrm{~d}$ at $\left.21{ }^{\circ} \mathrm{C}\right)$ simulated commercial storage.

\begin{tabular}{|c|c|c|c|c|c|c|c|c|c|}
\hline \multirow[b]{3}{*}{ Cultivar } & \multirow{3}{*}{$\begin{array}{c}\text { Storage } \\
\text { (d) }\end{array}$} & \multirow{2}{*}{\multicolumn{2}{|c|}{ 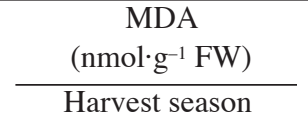 }} & \multirow{2}{*}{\multicolumn{2}{|c|}{$\begin{array}{c}\begin{array}{c}\text { Ascorbate } \\
\left(\mu \mathrm{mol} \cdot \mathrm{g}^{-1} \mathrm{FW}\right)\end{array} \\
\text { Harvest season }\end{array}$}} & \multirow{2}{*}{\multicolumn{2}{|c|}{$\begin{array}{c}\begin{array}{c}\text { } \text {-carotene } \\
\left(\mathrm{nmol}^{-\mathrm{g}^{-1}} \mathrm{FW}\right)\end{array} \\
\text { Harvest season }\end{array}$}} & \multirow{2}{*}{\multicolumn{2}{|c|}{$\begin{array}{c}\begin{array}{c}\text { Phenolics } \\
\left(\mathrm{mg} \cdot \mathrm{g}^{-1} \mathrm{FW}\right)\end{array} \\
\text { Harvest season }\end{array}$}} \\
\hline & & & & & & & & & \\
\hline & & Early & Late & Early & Late & Early & Late & Early & Late \\
\hline Cruiser & 0 & $1.8 \mathrm{~A}^{\mathrm{z}}, \mathrm{b}^{\mathrm{y}}$ & $1.3 \mathrm{~A}, \mathrm{~b}$ & $1.98 \mathrm{~A}, \mathrm{a}$ & $1.74 \mathrm{~A}, \mathrm{a}$ & $30.3 \mathrm{~B}, \mathrm{a}$ & $38.9 \mathrm{~B}, \mathrm{a}$ & $0.23 \mathrm{~A}, \mathrm{~b}$ & $0.25 \mathrm{~A}, \mathrm{~b}$ \\
\hline Honey Brew & 0 & $0.9 \mathrm{~A}, \mathrm{c}$ & $1.4 \mathrm{~A}, \mathrm{~b}$ & $1.91 \mathrm{~A}, \mathrm{a}$ & $1.37 \mathrm{~B}, \mathrm{a}$ & ND & ND & $0.25 \mathrm{~A}, \mathrm{~b}$ & $0.25 \mathrm{~A}, \mathrm{~b}$ \\
\hline Orange Dew & 0 & $0.3 \mathrm{~A}, \mathrm{c}$ & $1.6 \mathrm{~A}, \mathrm{~b}$ & $1.90 \mathrm{~A}, \mathrm{a}$ & $1.74 \mathrm{~A}, \mathrm{a}$ & $53.2 \mathrm{~A}, \mathrm{a}$ & $45.6 \mathrm{~A}, \mathrm{a}$ & $0.30 \mathrm{~A}, \mathrm{~b}$ & 0.29 A,b \\
\hline Cruiser & 10 & $10.2 \mathrm{~A}, \mathrm{a}$ & $9.6 \mathrm{~A}, \mathrm{a}$ & $1.96 \mathrm{~A}, \mathrm{a}$ & $1.48 \mathrm{~A}, \mathrm{a}$ & $32.1 \mathrm{~B}, \mathrm{a}$ & $35.9 \mathrm{~B}, \mathrm{a}$ & $0.24 \mathrm{~B}, \mathrm{~b}$ & $0.30 \mathrm{~B}, \mathrm{~b}$ \\
\hline Honey Brew & 10 & $10.9 \mathrm{~A}, \mathrm{a}$ & $6.2 \mathrm{~B}, \mathrm{a}$ & $1.44 \mathrm{~A}, \mathrm{~b}$ & $1.31 \mathrm{~A}, \mathrm{a}$ & ND & ND & $0.27 \mathrm{AB}, \mathrm{a}$ & $0.31 \mathrm{~B}, \mathrm{a}$ \\
\hline Orange Dew & 10 & $11.5 \mathrm{~A}, \mathrm{a}$ & $6.3 \mathrm{~B}, \mathrm{a}$ & $1.47 \mathrm{~A}, \mathrm{~b}$ & $1.30 \mathrm{~A}, \mathrm{~b}$ & $56.0 \mathrm{~A}, \mathrm{a}$ & 49.1 A,a & $0.31 \mathrm{~A}, \mathrm{~b}$ & $0.39 \mathrm{~A}, \mathrm{a}$ \\
\hline Cruiser & 17 & $7.5 \mathrm{~A}, \mathrm{a}$ & $8.2 \mathrm{~A}, \mathrm{a}$ & $1.64 \mathrm{~A}, \mathrm{a}$ & $1.49 \mathrm{~A}, \mathrm{a}$ & $32.0 \mathrm{~B}, \mathrm{a}$ & $30.3 \mathrm{~B}, \mathrm{a}$ & $0.35 \mathrm{~B}, \mathrm{a}$ & $0.37 \mathrm{~A}, \mathrm{a}$ \\
\hline Honey Brew & 17 & $5.3 \mathrm{~B}, \mathrm{~b}$ & $6.7 \mathrm{~B}, \mathrm{a}$ & $1.10 \mathrm{~B}, \mathrm{~b}$ & $1.21 \mathrm{~A}, \mathrm{a}$ & ND & ND & $0.32 \mathrm{~B}, \mathrm{a}$ & $0.31 \mathrm{~B}, \mathrm{a}$ \\
\hline Orange Dew & 17 & 4.4 B,b & $6.6 \mathrm{~B}, \mathrm{a}$ & $1.40 \mathrm{~B}, \mathrm{~b}$ & $1.22 \mathrm{~A}, \mathrm{~b}$ & $48.2 \mathrm{~A}, \mathrm{a}$ & $43.7 \mathrm{~A}, \mathrm{a}$ & $0.40 \mathrm{~A}, \mathrm{a}$ & $0.37 \mathrm{~A}, \mathrm{a}$ \\
\hline
\end{tabular}

${ }^{2}$ Capital letters within a column indicate significant differences $(P<0.05)$ among cultivars within a storage period.

ySmall letters within a column indicate significant differences $(P<0.05)$ within a cultivar over storage time.

Table 3. Ascorbate peroxidase (ASPX), monodehydroascorbate reductase (MDHAR), and dehydroascorbate reductase (DHAR) activities in orange-fleshed cantaloupe cultivar Cruiser, green-fleshed honeydew cultivar Honey Brew, and orange-fleshed honeydew cultivar Orange Dew fruits from plants at the beginning (Early) of the harvest season and from the same plants at the end of the harvest season (Late) and following $0,10\left(7 \mathrm{~d}\right.$ at $10{ }^{\circ} \mathrm{C}$ and $95 \%+2 \% \mathrm{RH}$ plus $3 \mathrm{~d}$ at $\left.21^{\circ} \mathrm{C}\right)$, or $17 \mathrm{~d}\left(14 \mathrm{~d}\right.$ at $10^{\circ} \mathrm{C}$ and $95 \%+2 \% \mathrm{RH}$ plus $3 \mathrm{~d}$ at $\left.21^{\circ} \mathrm{C}\right)$ simulated commercial storage.

\begin{tabular}{|c|c|c|c|c|c|c|c|}
\hline \multirow[b]{3}{*}{ Cultivar } & \multirow{3}{*}{$\begin{array}{c}\text { Storage } \\
\text { (d) }\end{array}$} & \multirow{2}{*}{\multicolumn{2}{|c|}{$\begin{array}{c}\text { ASPX } \\
\begin{array}{c}\text { ascorbate oxidized } \\
\left(\mathrm{nmol} \cdot \mathrm{g}^{-1} \mathrm{FW}\right)\end{array} \\
\text { Harvest season }\end{array}$}} & \multirow{2}{*}{\multicolumn{2}{|c|}{$\begin{array}{c}\text { MDHAR } \\
\begin{array}{c}\text { NADH oxidized } \\
\left(\mathrm{nmol}^{-\mathrm{g}^{-1}} \mathrm{FW}\right)\end{array} \\
\text { Harvest season }\end{array}$}} & \multirow{2}{*}{\multicolumn{2}{|c|}{$\begin{array}{c}\text { DHAR } \\
\begin{array}{c}\text { ascorbate reduced } \\
\left(\mathrm{mg} \cdot \mathrm{g}^{-1} \mathrm{FW}\right)\end{array} \\
\text { Harvest season }\end{array}$}} \\
\hline & & & & & & & \\
\hline & & Early & Late & Early & Late & Early & Late \\
\hline Cruiser & 0 & $4.17 \mathrm{C}^{\mathrm{z}}, \mathrm{a}^{\mathrm{y}}$ & $4.22 \mathrm{~B}, \mathrm{a}$ & $0.033 \mathrm{~A}, \mathrm{a}$ & $0.010 \mathrm{~B}, \mathrm{c}$ & $0.06 \mathrm{~B}, \mathrm{a}$ & $0.12 \mathrm{~B}, \mathrm{a}$ \\
\hline Honey Brew & 0 & $4.61 \mathrm{~B}, \mathrm{a}$ & $4.24 \mathrm{~B}, \mathrm{a}$ & $0.010 \mathrm{C}, \mathrm{c}$ & 0.014 B,b & $0.15 \mathrm{~A}, \mathrm{a}$ & $0.15 \mathrm{AB}, \mathrm{b}$ \\
\hline Orange Dew & 0 & $5.67 \mathrm{~A}, \mathrm{a}$ & $4.85 \mathrm{~A}, \mathrm{a}$ & $0.025 \mathrm{~B}, \mathrm{~b}$ & $0.021 \mathrm{~A}, \mathrm{~b}$ & $0.20 \mathrm{~A}, \mathrm{a}$ & $0.19 \mathrm{~A}, \mathrm{a}$ \\
\hline Cruiser & 10 & $4.24 \mathrm{~B}, \mathrm{a}$ & $3.23 \mathrm{~B}, \mathrm{a}$ & $0.015 \mathrm{C}, \mathrm{b}$ & $0.023 \mathrm{~B}, \mathrm{~b}$ & $0.05 \mathrm{~B}, \mathrm{a}$ & $0.10 \mathrm{~B}, \mathrm{~b}$ \\
\hline Honey Brew & 10 & $4.69 \mathrm{AB}, \mathrm{a}$ & 4.18 B,ab & $0.026 \mathrm{~B}, \mathrm{a}$ & $0.022 \mathrm{~B}, \mathrm{a}$ & $0.15 \mathrm{~A}, \mathrm{a}$ & $0.11 \mathrm{~B}, \mathrm{a}$ \\
\hline Orange Dew & 10 & $4.97 \mathrm{~A}, \mathrm{ab}$ & $4.83 \mathrm{~A}, \mathrm{a}$ & $0.036 \mathrm{~A}, \mathrm{a}$ & $0.028 \mathrm{~A}, \mathrm{~b}$ & $0.15 \mathrm{~A}, \mathrm{a}$ & $0.17 \mathrm{~A}, \mathrm{a}$ \\
\hline Cruiser & 17 & $3.69 \mathrm{~B}, \mathrm{a}$ & $3.20 \mathrm{~B}, \mathrm{a}$ & $0.023 \mathrm{~B}, \mathrm{~b}$ & $0.034 \mathrm{~A}, \mathrm{a}$ & $0.06 \mathrm{~B}, \mathrm{a}$ & $0.06 \mathrm{~B}, \mathrm{~b}$ \\
\hline Honey Brew & 17 & $4.27 \mathrm{~A}, \mathrm{a}$ & $4.14 \mathrm{~A}, \mathrm{~b}$ & $0.019 \mathrm{~B}, \mathrm{~b}$ & $0.026 \mathrm{~B}, \mathrm{a}$ & $0.08 \mathrm{~B}, \mathrm{a}$ & $0.09 \mathrm{~B}, \mathrm{a}$ \\
\hline Orange Dew & 17 & 4.70 A,b & $4.69 \mathrm{~A}, \mathrm{a}$ & $0.032 \mathrm{~A}, \mathrm{a}$ & $0.037 \mathrm{~A}, \mathrm{a}$ & $0.16 \mathrm{~A}, \mathrm{a}$ & $0.16 \mathrm{~A}, \mathrm{a}$ \\
\hline
\end{tabular}

${ }^{2}$ Capital letters within a column indicate significant differences $(P<0.05)$ among cultivars within a storage period.

ySmall letters within a column indicate significant differences $(P<0.05)$ within a cultivar over storage time.

Table 4. Glutathione reductase (GR), catalase (CAT), guaiacol peroxidase (POX), and superoxide dismutase (SOD) activities in orange-fleshed cantaloupe cultivar Cruiser, green-fleshed honeydew cultivar Honey Brew and orange-fleshed honeydew cultivar Orange Dew fruits from plants at the beginning (Early) of the harvest season and from the same plants at the end of the harvest season (Late) and following 0,10 ( $7 \mathrm{~d}$ at 10 ${ }^{\circ} \mathrm{C}$ and $95 \%+2 \% \mathrm{RH}$ plus $3 \mathrm{~d}$ at $\left.21{ }^{\circ} \mathrm{C}\right)$, or $17 \mathrm{~d}\left(14 \mathrm{~d}\right.$ at $10{ }^{\circ} \mathrm{C}$ and $95 \%+2 \% \mathrm{RH}$ plus $3 \mathrm{~d}$ at $\left.21{ }^{\circ} \mathrm{C}\right)$ simulated commercial storage.

\begin{tabular}{|c|c|c|c|c|c|c|c|c|c|}
\hline \multirow[b]{3}{*}{ Cultivar } & \multirow{3}{*}{$\begin{array}{c}\text { Storage } \\
\text { (d) }\end{array}$} & \multirow{2}{*}{\multicolumn{2}{|c|}{$\begin{array}{c}\text { GR } \\
\begin{array}{c}\text { NADPH oxidized } \\
\left(\mathrm{mmol} \cdot \mathrm{min}^{-1} \cdot \mathrm{g}^{-1} \mathrm{FW}\right)\end{array} \\
\text { Harvest season }\end{array}$}} & \multirow{2}{*}{\multicolumn{2}{|c|}{$\begin{array}{c}\text { CAT } \\
\begin{array}{c}\mathrm{H}_{2} \mathrm{O}_{2} \text { decomp. } \\
\left(\mathrm{mmol} \cdot \mathrm{min}^{-1} \cdot \mathrm{g}^{-1} \mathrm{FW}\right)\end{array} \\
\text { Harvest season }\end{array}$}} & \multirow{2}{*}{\multicolumn{2}{|c|}{$\begin{array}{c}\text { POX } \\
\begin{array}{c}\text { tetraguaiacol form. } \\
\left(\mathrm{mmol} \cdot \mathrm{min}^{-1} \cdot \mathrm{g}^{-1} \mathrm{FW}\right)\end{array} \\
\text { Harvest season }\end{array}$}} & \multirow{2}{*}{\multicolumn{2}{|c|}{$\begin{array}{c}\text { SOD } \\
\begin{array}{c}\text { cyt.c } \text { conserved } \\
\left(\mathrm{mmol} \cdot \mathrm{min}^{-1} \cdot \mathrm{g}^{-1} \mathrm{FW}\right)\end{array} \\
\text { Harvest season }\end{array}$}} \\
\hline & & & & & & & & & \\
\hline & & Early & Late & Early & Late & Early & Late & Early & Late \\
\hline Cruiser & 0 & $0.05 \mathrm{~A}^{\mathrm{z}}, \mathrm{a}^{\mathrm{y}}$ & $0.02 \mathrm{~B}, \mathrm{a}$ & $0.015 \mathrm{~B}, \mathrm{a}$ & $0.009 \mathrm{~B}, \mathrm{a}$ & $0.28 \mathrm{~B}, \mathrm{a}$ & $0.26 \mathrm{~B}, \mathrm{ab}$ & $0.015 \mathrm{~B}, \mathrm{a}$ & $0.017 \mathrm{AB}, \mathrm{a}$ \\
\hline Honey Brew & 0 & $0.02 \mathrm{~B}, \mathrm{a}$ & $0.01 \mathrm{~B}, \mathrm{a}$ & $0.033 \mathrm{~A}, \mathrm{a}$ & $0.030 \mathrm{~A}, \mathrm{a}$ & $0.29 \mathrm{~B}, \mathrm{a}$ & $0.49 \mathrm{~A}, \mathrm{a}$ & $0.054 \mathrm{~A}, \mathrm{a}$ & $0.015 \mathrm{~B}, \mathrm{~b}$ \\
\hline Orange Dew & 0 & $0.06 \mathrm{~A}, \mathrm{a}$ & $0.04 \mathrm{~A}, \mathrm{a}$ & $0.030 \mathrm{~A}, \mathrm{a}$ & $0.015 \mathrm{~B}, \mathrm{a}$ & $0.54 \mathrm{~A}, \mathrm{a}$ & $0.43 \mathrm{~A}, \mathrm{a}$ & $0.053 \mathrm{~A}, \mathrm{a}$ & $0.020 \mathrm{~A}, \mathrm{ab}$ \\
\hline Cruiser & 10 & $0.04 \mathrm{~A}, \mathrm{ab}$ & $0.02 \mathrm{~B}, \mathrm{a}$ & $0.015 \mathrm{~B}, \mathrm{a}$ & $0.011 \mathrm{~B}, \mathrm{a}$ & $0.32 \mathrm{~B}, \mathrm{a}$ & $0.23 \mathrm{~B}, \mathrm{~b}$ & $0.017 \mathrm{~B}, \mathrm{a}$ & $0.012 \mathrm{~B}, \mathrm{ab}$ \\
\hline Honey Brew & 10 & $0.02 \mathrm{~B}, \mathrm{a}$ & $0.02 \mathrm{~B}, \mathrm{a}$ & $0.013 \mathrm{~B}, \mathrm{~b}$ & $0.016 \mathrm{AB}, \mathrm{b}$ & $0.31 \mathrm{~B}, \mathrm{a}$ & $0.47 \mathrm{~A}, \mathrm{a}$ & $0.023 \mathrm{~A}, \mathrm{~b}$ & $0.014 \mathrm{AB}, \mathrm{b}$ \\
\hline Orange Dew & 10 & $0.05 \mathrm{~A}, \mathrm{ab}$ & $0.03 \mathrm{~A}, \mathrm{a}$ & $0.025 \mathrm{~A}, \mathrm{a}$ & $0.023 \mathrm{~A}, \mathrm{a}$ & $0.46 \mathrm{~A}, \mathrm{~b}$ & $0.44 \mathrm{~A}, \mathrm{a}$ & $0.028 \mathrm{~A}, \mathrm{~b}$ & $0.018 \mathrm{~A}, \mathrm{~b}$ \\
\hline Cruiser & 17 & $0.02 \mathrm{~A}, \mathrm{~b}$ & $0.02 \mathrm{~B}, \mathrm{a}$ & $0.012 \mathrm{~B}, \mathrm{a}$ & $0.012 \mathrm{~B}, \mathrm{a}$ & $0.26 \mathrm{~B}, \mathrm{a}$ & $0.32 \mathrm{~B}, \mathrm{a}$ & $0.015 \mathrm{~B}, \mathrm{a}$ & $0.009 \mathrm{~B}, \mathrm{~b}$ \\
\hline Honey Brew & 17 & $0.01 \mathrm{~B}, \mathrm{~b}$ & $0.01 \mathrm{~B}, \mathrm{a}$ & $0.006 \mathrm{~B}, \mathrm{~b}$ & $0.013 \mathrm{~B}, \mathrm{~b}$ & $0.29 \mathrm{~B}, \mathrm{a}$ & $0.44 \mathrm{~A}, \mathrm{a}$ & $0.019 \mathrm{AB}, \mathrm{b}$ & $0.022 \mathrm{~A}, \mathrm{a}$ \\
\hline Orange Dew & 17 & $0.03 \mathrm{~A}, \mathrm{~b}$ & $0.04 \mathrm{~A}, \mathrm{a}$ & $0.021 \mathrm{~A}, \mathrm{a}$ & $0.016 \mathrm{~A}, \mathrm{a}$ & $0.43 \mathrm{~A}, \mathrm{~b}$ & $0.48 \mathrm{~A}, \mathrm{a}$ & $0.021 \mathrm{~A}, \mathrm{~b}$ & $0.024 \mathrm{~A}, \mathrm{a}$ \\
\hline
\end{tabular}

${ }^{\mathrm{z}}$ Capital letters within a column indicate significant differences $(P<0.05)$ among cultivars within a storage period.

ySmall letters within a column indicate significant differences $(P<0.05)$ within a cultivar over storage time. 
of this enzyme were noted between 'Orange Dew' and 'Honey Brew' on these days (Table 4). Although a dramatic decline during storage was observed in SOD activities of both non-netted muskmelons from the fruit harvested a the start of the harvest season, no such changes were noted in fruit from the end of the harvest season.

The first, second, and third PCA for the enzymes and MDA accounted for $36.0 \%, 20.3 \%$, and $14.6 \%$ of the variation, respectively. The first principal component showed that, in general, both 'Orange Dew' and 'Honey Brew' had more negative scores than 'Cruiser' (data not shown). The negative scores for 'Orange Dew' and 'Honey Brew' were weighted by the means of SOD, AsPX, DHAR, CAT, and POX activities. The scores of the third principal component were only negative for 'Cruiser,' and these scores were weighted by SOD and AsPX activities.

Total ANTIOXIDANTS. No overall change in total hydrophilic antioxidant levels (Trolox equivalents per gram DW) within cultivars during storage were noted (Fig. 1A). No differences in total hydrophilic antioxidant content were observed between the

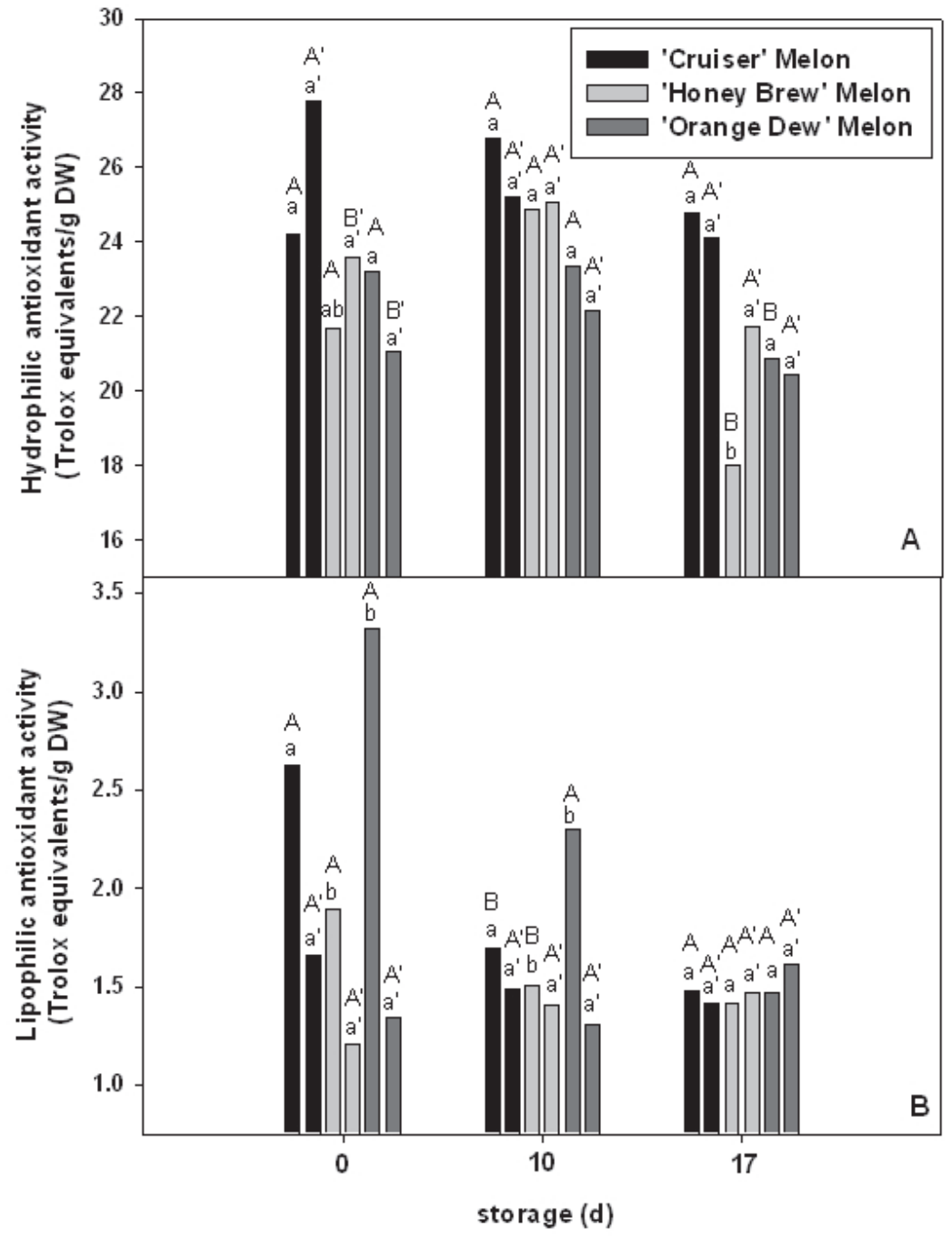

Fig. 1. Total hydrophilic (A) and lipophilic (B) antioxidant activities in orange-fleshed cantaloupe cultivar Cruiser, green-fleshed honeydew cultivar Honey Brew, and orange-fleshed honeydew cultivar Orange Dew fruit from plants at the beginning of the harvest season (early; without ') and from the same plants at the end of the harvest season (late; with ') and following 0,10 (7 d at $10{ }^{\circ} \mathrm{C}$ and $95 \% \pm 2 \% \mathrm{RH}$ plus $3 \mathrm{~d}$ at $\left.21{ }^{\circ} \mathrm{C}\right)$ or $17 \mathrm{~d}\left(14 \mathrm{~d}\right.$ at $10{ }^{\circ} \mathrm{C}$ and $95 \% \pm 2 \% \mathrm{RH}$ plus 3 $\mathrm{d}$ at $21{ }^{\circ} \mathrm{C}$ ) simulated commercial storage. Capital letters within a column indicate significant differences $(P \leq 0.05)$ among cultivars within a storage period, whereas small letters within a column indicate significant differences $(P \leq 0.05)$ within a cultivar over storage time. three cultivars with the exception of days 17 and 0 of the early and late harvest, respectively, where the water-soluble antioxidant activity of the netted 'Cruiser' was higher than that of both 'Orange Dew' and 'Honey Brew'. There were no significant differences in hydrophilic antioxidant activity in fruit between the start or the end of the harvest season (Table 1).

The only differences in lipophilic antioxidant activity (Trolox equivalents per gram DW) between the cultivars occurred on day 10 in fruit from the start of the harvest season, where the lipidsoluble antioxidant activity of 'Orange Dew' was higher than either 'Cruiser' or 'Honey Brew' (Fig. 1B). The lipophilic antioxidant activity was lower on days 0 and 10 in both 'Orange Dew' and oney Brew' cultivars at the start of the harvest season, but no differences were observed between cultivars or within cultivars in fruit at the end of the harvest season.

\section{Discussion}

In comparison with the netted muskmelon 'Cruiser,' the nonnetted orange-fleshed muskmelon 'Orange Dew' exhibited higher $(15 \%$ to $43 \%)$ concentrations of $\beta$-carotene and, with few exceptions, higher activities of the antioxidant enzymes AsPX, MDHAR, DHAR, CAT, POX, and SOD. These finding, observed on day 0 and following $17 \mathrm{~d}$ simulated commercial/retail storage, suggest that 'Orange Dew' has an inherently greater potential for antioxidant capacity than does the netted 'Cruiser'. The lower levels of MDA, an estimator of lipid peroxidation (Hodges et al., 1999), in 'Orange Dew' also indicates that the greater antioxidant capacity of this cultivar had an effect on reducing AOS levels and, hence, oxidative stress, compared to 'Cruiser'.

Phenolics play an important role in fruit flavor, color, and regulation of senescence by contributing to antioxidant activity and oxidative stability (Del Carlo et al., 2004). Though phenolics in fruit of all three cultivars increased with storage duration, 'Orange Dew' generally exhibited higher levels of phenolics versus 'Cruiser' or 'Honey Brew' on day 10 and day 17.

Presumably, higher activities of SOD in 'Orange Dew' led to greater $\mathrm{O}_{2}$ - dismutation and subsequent $\mathrm{H}_{2} \mathrm{O}_{2}$ production than in 'Cruiser' (for review of antioxidants, see Lurie, 2003). The higher activities of CAT, POX, and AsPX in 'Orange Dew', perhaps in response to the higher SOD activities, would give this cultivar an advantage in converting $\mathrm{H}_{2} \mathrm{O}_{2}$ from SOD activity as well as from other sources to $\mathrm{H}_{2} \mathrm{O}$ as compared to 'Cruiser'. As ascorbate is oxidized to dehydroascorbate in the reaction catalyzed by AsPX, this would have necessitated the presence in 'Orange Dew' of higher activities of MDHAR and DHAR, which re-reduce dehydroascorbate back to ascorbate.

Both 'Orange Dew' and 'Honey Brew' exhibited lower lipid peroxidation levels, as estimated by MDA content, than 'Cruiser' during storage. However, only ASPX and SOD activities of 'Honey Brew' were similar to those of 'Orange Dew'; activities of MDHAR, DHAR, CAT, and POX were, for the most part, no different than those of 'Cruiser'. This 
suggests that the critical antioxidant enzymes for regulating postharvest-induced oxidative stress in non-netted muskmelon are AsPX and SOD, and that MDHAR, GR, DHAR, CAT, POX play secondary roles. The similarities in lipid peroxidation during storage between the two non-netted honey dew (green- and orange-fleshed) cultivars suggests that $\beta$-carotene may play a less obvious role in limiting oxidative stress during postharvest melon storage.

In a previous study comparing CAT, POX, and SOD activities between two cultivars of non-netted muskmelon fruit ('Clipper') and ('Jerac') differing in their storage life 'Clipper,' the cultivar which exhibited less rapid lipid peroxidation(i.e., lower senescence rate) and AOS proliferation than 'Jerac', also had higher initial activities levels of CAT and SOD and maintained higher activities of these enzymes throughout a 14-d storage period (Lacan and Baccou, 1998). Although initial values for POX were similar for the two cultivars, and activities of this enzyme decreased during storage, POX activity in 'Jerac' declined more rapidly. The ascorbate-related antioxidant enzymes were not assessed in the Lacan and Baccou (1998) study.

A decline in activities of SOD and ASPX of fresh red peppers during a 19-d storage period corroborate that these antioxidant enzymes contribute to the control of postharvest senescence and quality loss (Jiménez et al., 2003). After studying a number of antioxidant enzymes and compounds, activities of ASPX and SOD and concentrations of ascorbate were singled out as being important in the dynamics and severity of postharvest quality loss in fresh-cut spinach (Hodges et al., 2001; Hodges and Forney, 2000, 2003). Similar to the present muskmelon study, ASPX activities declined far more rapidly in the leaves of a spinach cultivar which accumulated more MDA than another cultivar during a 14-d storage period (Hodges et al., 2001). However, in contrast to the muskmelon results, SOD activity decreased in the cultivar which exhibited greater MDA accumulation. As with ASPX activities, spinach leaf concentrations of ascorbate declined more rapidly in the cultivar that demonstrated the greatest potential for lipid peroxidation. Surprisingly, there were no significant differences in total ascorbate concentrations between netted orange-fleshed muskmelon 'Crusier' and non-netted orange-fleshed honeydew 'Orange Dew' in this study. However, the higher capacity to cycle ascorbate through the $\mathrm{H}_{2} \mathrm{O}_{2}$ removal pathway possessed by 'Orange Dew' and 'Honey Brew', as represented by higher ASPX activities, may have provided these non-netted cultivars an advantage over 'Cruiser' in neutralizing this particular AOS, which was independent upon the actual concentration of ascorbate.

The reason why the activities of the antioxidant enzymes ASPX, CAT, MDHAR, DHAR, POX, and SOD are initially and remain higher in 'Orange Dew' than 'Cruiser' remain unclear. As AOS can act as secondary cellular signals which induce apparently co-regulated antioxidant defenses (e.g., Shigeoka et al., 2002; Starzyńska et al., 2003; Vranová et al., 2002), it is possible that 'Orange Dew' either inherently produces higher amounts of AOS, is more sensitive to changes in AOS levels, and/or contains a more efficient cellular signal-gene expression system. In contrast to our results, mutants of arabidopsis [Arabidopsis thaliana (L.) Heynh.] exhibiting delayed leaf senescence and increased tolerance to oxidative stress evinced AsPX, CAT, and SOD activities which were similar or slightly lower than those of wild-type plants under unstressed conditions; the addition of methyl viologen to stimulate oxidative stress increased activities of these antioxidant enzymes to a lesser degree than in the wild type (Woo et al., 2004). Woo et al. (2004) concluded that increased tolerance to oxidative stress in the delayed leaf senescence of arabidopsis mutants did not appear to be associated with enhanced activities of AsPX, CAT, or SOD. However, as transgenic plants overexpressing antioxidant enzyme activities such as AsPX and SOD have shown a concomitant increase in tolerance to stress-and/or senescence induced oxidative stress in other plant systems (e.g., Wang et al., 2005; Yabuta et al., 2002), it appears that the role of antioxidants in senescence is very situation dependent.

The greater antioxidant potential of 'Orange Dew' as measured by $ß$-carotene and phenolic content and certain enzyme activities was not reflected in the AAPH-based hydrophilic or lipophilic total antioxidant activity assays. These total antioxidant assay would not, of course, directly estimate enzyme-related antioxidant potential. Moreover, equivalent concentrations of $ß$-carotene provided $\approx 5 \%$ of the total lipophilic antioxidant activity values as did $\alpha$-tocopherol using this AAPH assay system, which is not surprising as Trolox is a water-soluble $\alpha$-tocopherol analogue (personal observation). Recent findings demonstrate that the concentration of RMCD is highly critical in correlating levels of lycopene (another carotenoid) with total antioxidant activity, and increasing the percentage RMCD led to a decrease in overall lycopene-related Trolox equivalent values (Bangalore et al., 2005). The RMCD concentration used in our study, following the Prior et al. (2003) protocol, likely was too high, leading to underestimation of the total antioxidant activity contribution from B-carotene. Furthermore, carotenoids can act as potent antioxidants in lipid-rich environments (Kiokias and Gordon, 2004). There is evidence that ascorbic acid can inhibit carotenoid antioxidant activity in water model systems, but mixtures of carotenoids with ascorbic acid exhibit enhanced antioxidant activity with oil-water emulsions (Kiokias and Gordon, 2004). Although the greater B-carotene contents realized in 'Orange Dew' did not generate heightened lipophilic antioxidant activities using current methodologies (Prior et al., 2003), the 1.2- to 1.8-fold higher levels of $\beta$-carotene in non-netted 'Orange Dew' vs. netted 'Cruiser' nevertheless will make 'Orange Dew' more appealing to consumers (Lester and Turley, 1990). Additionally, higher levels of $ß$-carotene per "serving" of melon would provide consumers with heightened anti-cancer, heart disease, stroke, cardiovascular disease, chronic fatigue, and night blindness health benefits (Lester, 1997) as there is sound epidemiological evidence linking higher ingestion of carotene-containing food matrixes with reduced incidences of certain forms of cancer and cardiovascular disease (Kiokias and Gordon, 2004).

Additional research into comparisons of 'Orange Dew' vs. 'Cruiser' needs to consider the impact of growing environment on enzymatic and nonenzymatic antioxidants. It is known that phytonutrients (e.g., ascorbic acid, $\beta$-carotene, and folic acid) accumulate differently in various cultivars of netted muskmelons and of honey dew melons in response to different growing locations, and yearly conditions (Lester and Crosby, 2002; Lester and Eischen, 1996). Likely, enzymatic and non-enzymatic antioxidant contents will also vary in 'Orange Dew' vs. 'Cruiser' melons grown under different environmental conditions.

In conclusion, due to the lack of a netted rind, non-netted orange-fleshed honey dew ('Orange Dew') does not represent the risk to consumers as does netted muskmelon ('Cruiser'), as human illness pathogens can remain present in netting inaccessible to sanitation. Furthermore, both orange-fleshed ('Orange Dew') and green-fleshed ('Honey Brew') non-netted cultivars evinced less lipid peroxidation, and hence postharvest senescence, during the 17-d storage period then the netted muskmelon 'Cruiser'. 
Higher AsPX and SOD activities in 'Orange Dew' and 'Honey Brew' appear to confer a greater resistance to lipid peroxidation in these muskmelon genotypes than in the netted 'Cruiser'. Other studies have also identified AsPX and SOD as enzymes playing an important role in postharvest oxidative stress of fruit and vegetables (e.g., Hodges et al., 2001; Jiménez et al., 2003). Although 'Orange-Dew' contains relatively high amounts of $\beta$ carotene [approximately one-third the $57.7 \mu \mathrm{g}^{\cdot} \mathrm{g}^{-1} \mathrm{FW}$ found in raw carrot (Daucus carota L.) according to the USDA National Nutrient Database (USDA, 2004)], levels of this antioxidant were undetectable in 'Honey Brew'. As the level of lipid peroxidation is similar between 'Orange Dew' and 'Honey Brew,' B-carotene thus appears to play little role in regulating postharvest oxidative stress and resultant quality loss. 'Orange Dew' appears to be a healthier food choice not only due to its lack a netted rind which could potentially harbour human illness-related pathogens, but also that it is superior to both 'Cruiser' and 'Honey Brew' in overall $\beta$-carotene and phenolic levels.

\section{Literature Cited}

Annous, B.A., A. Burke, and J.E. Sites. 2004. Surface pasteurization of whole fresh cantaloupes inoculated with Salmonella Poona or Escherichia coli. J. Food Protection 67:1876-1885.

Bangalore, D.V., W. McGlynn, and D.D. Scott. 2005. Effect of B-cyclodextrin in improving the correlation between lycopene and concentration and ORAC values. J. Agr. Food Chem. 53:1878-1883.

Barden, C.L. and W.J. Bramlage. 1994. Acclimation of antioxidants in apple peel as related to preharvest factors and superficial scald susceptibility of the fruit. J. Amer. Soc. Hort. Sci. 119:264-269.

Beuchat, L.R. and A.J. Scouten. 2004. Factors affecting survival, growth, and retrieval of Salmonella Poona in intact and wounded cantaloupe rind and stem scar tissue. Food Microbiol. 21:683-694.

Blokhina, O., E. Virolainen, and K.V. Fagerstedt. 2003. Antioxidants, oxidative damage and oxygen deprivation stress: A review. Ann. Bot. 91:179-194.

Bradford, M.M. 1976. A rapid and sensitive method for the quantification of microgram quantities of protein using the principle of protein dye-binding. Anal. Biochem. 72: 248-254.

Canadian Food Inspection Agency. 2003. Import requirements for Mexican cantaloupes. 12 Feb. 2005. http://www.inspection.gc.ca/english/plaveg/fresh/mexicane.shtml>.

Castillo, A., I. Mercado, I.M. Lucia, Y. Martinez-Ruiz, J. Ponce de Leon, E.A. Murano and G.R. Acuff. 2004. Salmonella contamination during production of cantaloupe: A binational study. J. Food Protection 67:713-720.

Del Carlo, M., G. Sacchetti, C. Di Mattia, D. Compagnone, D. Mastrocola, L. Liberatore, and A. Cichelli. 2004. Contribution of the phenolic fraction to the antioxidant activity and oxidative stability of olive oil. J. Agr. Food Chem. 52: 4072-4079.

Del Rosario, B.A. and L.R. Beuchat. 1995. Survival and growth of enterohemorrhagic Escherichia coli O157:H7 in cantaloupe and watermelon. J. Food Protection 58:105-107.

Edwards, M.E. and R.M. Blennerhassett. 1990. The use of postharvest treatments to extend storage life and to control postharvest wastage of honeydew melons (Cucumis melo var. indorus naud.) in cool storage. Austral. J. Expt. Agr. 30:693-698.

Hodges, D.M., J.M. DeLong, C.F. Forney, and R.K. Prange. 1999. Improving the thiobarbituric acid-reactive-substances assay for estimating lipid peroxidation in plant tissues containing anthocyanin and other interfering compounds. Planta 207:604-611.

Hodges, D.M. and C.F. Forney. 2000. The effects of ethylene, depressed oxygen and elevated carbon dioxide on antioxidant profiles of senescing spinach leaves. J. Expt. Bot. 51:645-655.

Hodges, D.M., W.V. Wismer, and C.F. Forney. 2001. Antioxidant responses in postharvest leaves of two cultivars of spinach (Spinacia oleracea L.) differing in their senescence rates. J. Amer. Soc. Hort. Sci. 126:611-617.
Hodges, D.M. and C.F. Forney. 2003. Postharvest ascorbate metabolism in two cultivars of spinach differing in their senescence rates. J. Amer. Soc. Hort. Sci. 128:930-935.

Hodges, D.M. 2003. Overview: Oxidative stress and postharvest produce, p. 1-12. In: D.M. Hodges (ed.). Postharvest oxidative stress in horticultural crops. Food Products Press, New York.

Hodges, D.M. and W. Kalt. 2003. Health functionality of small fruit. Acta Hort. 626:17-23.

Hodges, D.M., G.E. Lester, K.D. Munro, and P.M.A. Toivonen. 2004. Oxidative stress: Importance for postharvest quality. HortScience 39:924-929.

Horemans, N., C.H. Foyer, G. Potters, and H. Asard. 2000. Ascorbate function and associated transport systems in plants. Plant Physiol. Biochem. 38:531-540.

Huang, D., B. Ou, and R.L. Prior. 2005. The chemistry behind antioxidant capacity assays. J. Agr. Food Chem. 53:1841-1856.

Jiménez,A., F. Romojaro, J.M. Gómez, M.R. Llanos, and F. Sevilla. 2003. Antioxidant systems and their relationship with the response of pepper fruits to storage at $20^{\circ} \mathrm{C}$. J. Agr. Food Chem. 51:6293-6299.

Kiokias, S. and M.H. Gordon. 2004. Antioxidant properties of carotenoids in vitro and in vivo. Food Rev. Intl. 20:99-121.

Koch, T.C. and I.L. Goldman. 2004. Aone-pass semi-quantitative method for extraction and analysis of carotenoids and tocopherols in carrots. HortScience 39:1260-1261.

Knox, J.P. and A.D. Dodge. 1985. Singlet oxygen and plants. Phytochemistry 24:889-896.

Lacan, D. and J.-C. Baccou. 1998. High levels of antioxidant enzymes correlates with delayed senescence in non-netted muskmelon fruit. Planta 204:377-382.

Lester, G.E. 1988. Comparison of 'honey dew' and netted muskmelon fruit tissues in relation to storage life. HortScience 23:180-182.

Lester, G.E. and R. M. Turley. 1990. Chemical, physical and sensory comparisons of netted muskmelon fruit cultivars and breeding lines at harvest. J. Rio Grande Valley Hort. Soc. 43:71-77.

Lester, G.E. and F. Eischen. 1996. Beta-carotene content of postharvest orange-flesh muskmelon fruit: effect of cultivar, growing location, and fruit size. Plant Foods Human Nutr. 49:191-197

Lester, G. 1997. Melon (Cucumis melo L.) fruit nutritional quality and health functionality. HortTechnology 7:222-227.

Lester, G.E. and K. Crosby. 2002. Ascorbic acid, folic acid and potassium content in postharvest green-flesh honeydew muskmelons: Influence of cultivar, fruit size, soil type, and year. J. Amer. Soc. Hort. Sci. 127:843-847.

Lester, G., D.M. Hodges, R.D. Meyer, and K.D. Munro. 2004. Pre-extraction preparation (fresh, frozen, freeze-dried, or acetone powdered) and long-term storage of fruit and vegetable tissues: Effects on antioxidant enzyme activity. J. Agr. Food Chem. 52:2167-2173.

Lurie, S. 2003. Antioxidants, p. 131-150. In: D.M. Hodges (ed.). Postharvest oxidative stress in horticultural crops. Food Products Press, New York.

Olsson, M.E., J. Ekvall, K.-.E. Gustavsson, J. Nilsson, D. Pillai, I. Sjöholm, U. Svensson, B. Åkesson, and M.G.L. Nyman. 2004. Antioxidants, low molecular weight carbohydrates, and total antioxidant capacity in strawberries (Fragaria xananassa): Effects of cultivar, ripening, and storage. J. Agr. Food Chem. 52:2490-2498.

Prior, R.L., H. Hoang, L. Gu, X. Wu, M. Bacchiocca, L. Howard, M. Hampsch-Woodill, D. Hunag, B. Ou, and R. Jacob. 2003. Assays for hydrophilic and lipophilic antioxidant capacity (oxygen radical absorbance capacity $\left(\left(\mathrm{ORAC}_{\mathrm{FL}}\right)\right)$ of plasma and other biological and food samples. J. Agr. Food Chem. 51:3273-3279.

Proestos, C., N. Chorianopoulos, G.-J.E. Nychas, and M. Komaitis. 2005. RP-HPLC analysis of the phenolic compounds of plant extracts. Investigation of their antioxidant capacity and antimicrobial activity. J. Agr. Food Chem. 53:1190-1195.

Shigeoka, S., T. Ishikawa, M. Tamoi, Y. Miyagawa, T. Takeda, Y. Yabuta, and K. Yoshimura. 2002. Regulation and function of ascorbate peroxidase isoenzymes. J. Expt. Bot. 53:1305-1319.

Singleton, V.L. and J.A. Rossi. 1965. Colorimetry of total phenolics 
with phosphomolybdic- phosphotungstic acid. Amer. J. Enol. Viticult. 16:144-158.

Starzyńska, A., M. Leja, and A. Mareczek. 2003. Physiological changes in the antioxidant system of broccoli flower buds senescing during short-term storage, related to temperature and packaging. Plant Sci. 165:1387-1395.

Toivonen, P.M.A. and M. Sweeney. 1998. Differences in chlorophyll loss at $13{ }^{\circ} \mathrm{C}$ for two broccoli (Brassica oleracea L.) cultivars associated with antioxidant enzyme activities. J. Agr. Food Chem. 46:20-24.

Ukuku,D.O. 2004. The effect of hydrogen peroxide treatment on microbial quality and appearance of whole and fresh-cut melons contaminated with Salmonella spp. Intl. J. Food Microbiol. 95:137-146.

U.S. Department of Agriculture. 2004. USDANational Nutrient Database. 28 June 2005. <http: //www.nal.usda.gov/fnic/foodcomp/.

U.S. Food and Drug Administration. 2003. FDA survey of domestic fresh produce. 28 June $20005<$ http://www.cfsan.fda.gov>.

Vanoli, M., C. Visai, M.Zini, and R. Budini. 1995. Enzymatic activities and internal browning of Passa Crassana pears. Acta Hort. 379:405-411.

Vinson, J.A., X. Su, L. Zubik, and P. Bose. 2001. Phenol antioxidant quantity and quality in foods: fruits. J. Agr. Food Chem. 49:5315-5321.
Vranová, E., D. Inzé, and F. Van Breusegem. 2002. Signal transduction during oxidative stress. J. Expt. Bot. 53:1227-1236.

Wang, Y., M. Wisniewski, R. Melian, M. Cui, R. Webb, and L. Fuchigami. 2005. Overexpression of cytosolic ascorbate peroxidase in tomato confers tolerance to chilling and salt stress. J. Amer. Soc. Hort. Sci. 130:167-173.

Woo, H.R., J.H. Kim, H.G. Nam, and P.O. Lim. 2004. The delayed leaf senescence mutants of Arabidopsis, ore 1, ore 3, and ore 9 are tolerant to oxidative stress. Plant Cell Physiol. 45:923-932.

Yabuta, Y., T. Motoki, K. Yoshimura, T. Takeda, T. Ishikawa, and S. Shigeoka. 2002. Thylakoid membrane-bound ascorbate peroxidase is a limiting factor of antioxidative systems under photo-oxidative stress. Plant J. 32:915-925.

Yamauchi, N. and A. Kusabe. 2001. Involvement of ascorbate-glutathione cycle in senescence of stored broccoli (Brassica oleracea L.). J. Jpn. Soc. Hort. Sci. 70:704-708.

Zabrouskov, V., G.N.M. Kumar, J.P. Spychalla, and N.R. Knowles. 2002. Oxidative metabolism and the physiological age of seed potatoes are affected by increased alpha-linolenate content. Physiol. Plant. 116:172-185. 